\title{
Erratum: Phase-Slip Centers in Superconducting Indium Microbridges*
}

\author{
H. Weissbrod, R. P. Huebener, and W. Clauss \\ Physikalisches Institut II, Universität Tübingen, D-7400 Tübingen, West Germany
}

In Fig. 7 the quasiparticle diffusion length $\Lambda$ of the SBT model is mistakenly compared with the normal-conducting length from the KR theory. Actually, the latter length (also denoted $\Lambda$ by KR) is twice the value of the SBT quasiparticle diffusion length. As a consequence, our experimental points in Fig. 7 must be shifted upward by a factor of 2 .

In calculating the temperature-dependent coherence length $\xi(T)$, we used the dirty-limit expression (13). However, for the samples investigated the more general equation ${ }^{1}$

$$
\xi(T)=0.74\left[1+0.754\left(\xi_{0} / l\right)\right]^{-1 / 2} \xi_{0}\left(1-T / T_{c}\right)^{-1 / 2}
$$

appears to be preferable. In Fig. 7 this results in an additional shift of the experimental points upward by a factor of about 1.8 .

\section{REFERENCE}

1. R. Tidecks and G. Slama, Z. Phys. B 37, 103 (1980).

*This paper appeared in J. Low Temp. Phys. 69, 77 (1987). 